\title{
Use of oxygenate blends as inflammation aid in diluted mixtures
}

Moritz Grüninger, Dr. Olaf Toedter, Prof. Dr. sc. Techn. Thomas Koch

Institut für Kolbenmaschinen, Karlsruher Institut für Technologie

This manuscript is not available according to publishing restriction. Thank you for your understanding. 